\title{
The Finescale Response of Lowered ADCP Velocity Measurements Processed with Different Methods
}

\author{
A. M. THURNHERR \\ Lamont-Doherty Earth Observatory, Palisades, New York
}

(Manuscript received 16 September 2011, in final form 30 January 2012)

\begin{abstract}
In a paper published in 2002 in this journal, K. Polzin et al. derive corrections for spectra of vertical shear calculated from lowered acoustic Doppler current profiler (LADCP) velocity data. To illustrate and validate the corrections, they use velocities derived with a specific implementation of the shear method for LADCP processing that is no longer supported or widely used. In several recent publications, spectral corrections specific to this old processing method have been applied without modification to LADCP data processed with the more modern and much more widely used velocity-inversion method, which is associated with significantly less damping at high vertical wavenumbers than the older method. The purpose of this work is to derive and validate spectral corrections appropriate for different LADCP processing methods.
\end{abstract}

\section{Introduction}

In their paper, Polzin et al. (2002) compare spectra of vertical shear of horizontal velocity measured with a lowered acoustic Doppler current profiler (LADCP) to simultaneously collected expendable current profiler (XCP) shear spectra. They show that the LADCP-derived shear spectra are strongly damped at high vertical wavenumbers, and they derive spectral corrections to compensate for damping associated with velocity measurements collected with current Teledyne/RDI broadband ADCPs and with various data processing steps. The spectral corrections for LADCP shear data are particularly useful in the context of studying oceanic turbulence and mixing from CTD/LADCP finestructure data (e.g., NaveiraGarabato et al. 2004; Kunze et al. 2006; MacKinnon et al. 2008; Tian et al. 2009).

There are currently two fundamentally different methods for obtaining horizontal velocities from LADCP data, which are called the shear method (Firing and Gordon 1990; Fischer and Visbeck 1993; King et al. 2001) and the velocity-inversion method (Visbeck 2002). Publicly available implementations of both methods yield velocity profiles that are very similar in their lowvertical-mode content (Thurnherr 2010). On the other

Corresponding author address: A. M. Thurnherr, Lamont-Doherty Earth Observatory, P.O. Box 1000, Palisades, NY 10964-1000.

E-mail: ant@ldeo.columbia.edu hand, comparison of vertical-shear spectra calculated from the LADCP data of Polzin et al. (2002) processed with different methods reveals that, although all LADCP solutions are strongly damped at vertical wavelengths shorter than about $200 \mathrm{~m}$, the amount of damping depends significantly on the processing method (Fig. 1): the shear-method implementation developed at the University of Hawaii (UH) and described by King et al. (2001) (red); a modified implementation of the shear method, where gridding of the shear samples is accomplished by simple depth binning instead of interpolation (green); and the original implementation of the velocity-inversion method developed by Visbeck (2002) and currently maintained by this author at LDEO (blue). Because a 5-m vertical resolution for processing is hard coded in the UH shear-method implementation, the results presented here have all been processed at the same resolution for consistency.

\section{Spectral corrections for different LADCP processing methods}

Ignoring beam-separation effects by setting $T_{\mathrm{bsl}}=1$ as suggested by Polzin et al. (2002), the spectral correction (transfer function) for vertical shear derived from LADCP data processed with the $\mathrm{UH}$ implementation of the shear method becomes

$$
T_{\mathrm{UH}}=T_{\text {ra }} \times T_{\text {fd }} \times T_{\text {int }} \times T_{\text {tilt }},
$$




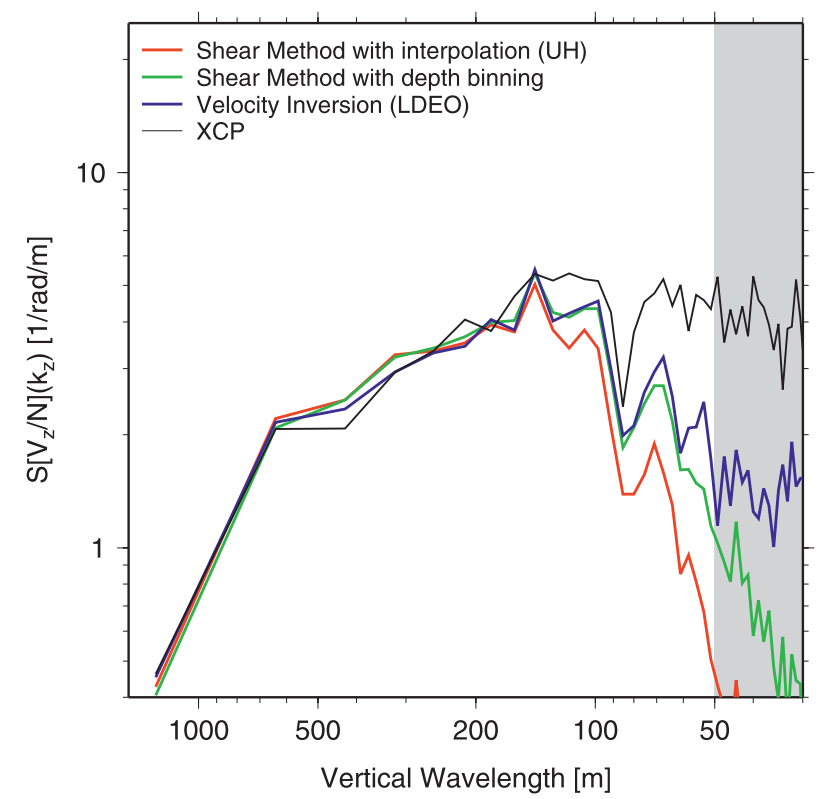

FIG. 1. Vertical wavenumber spectra of buoyancy-frequencynormalized vertical shear between 220 and $1500 \mathrm{~m}$ in the dataset of Polzin et al. (2002); the wavenumber range not resolved by the $\operatorname{LADCP}\left(\lambda_{z} \leq 50 \mathrm{~m}\right)$ is shaded gray; each spectral curve represents an ensemble average derived from 18 profiles; as in Polzin et al. (2002), parabolic fits were removed from the data before calculating the spectra. The red, green, and blue curves are derived from LADCP data processed at 5-m vertical resolution with three different methods (see text for details); the black curve shows the corresponding XCP-derived shear spectrum.

where the symbols are as in Polzin et al. (2002); that is, $T_{\text {ra }}, T_{\text {fd }}, T_{\text {int }}$, and $T_{\text {tilt }}$ denote the spectral transfer functions to correct for range averaging, finite differencing, shear interpolation, and instrument tilting, respectively,

$$
\begin{aligned}
& T_{\mathrm{ra}}\left(k_{z}\right)=\operatorname{sinc}^{2}\left(\frac{k_{z} \Delta z_{t}}{2 \pi}\right) \operatorname{sinc}^{2}\left(\frac{k_{z} \Delta z_{r}}{2 \pi}\right), \\
& T_{\mathrm{fd}}\left(k_{z}\right)=\operatorname{sinc}^{2}\left(\frac{k_{z} \Delta z_{r}}{2 \pi}\right), \\
& T_{\mathrm{int}}\left(k_{z}\right)=\operatorname{sinc}^{4}\left(\frac{k_{z} \Delta z_{r}}{2 \pi}\right) \operatorname{sinc}^{2}\left(\frac{k_{z} \Delta z_{g}}{2 \pi}\right), \text { and } \\
& T_{\text {tilt }}\left(k_{z}\right)=\operatorname{sinc}^{2}\left(\frac{k_{z} d^{\prime}}{2 \pi}\right) .
\end{aligned}
$$

Here, $k_{z}$ is the vertical wavenumber, $\Delta z_{r}$ the ADCP bin length, $\Delta z_{t}$ the ADCP transmit pulse length (usually $\left.\Delta z_{t}=\Delta z_{r}\right), \Delta z_{g}$ the vertical resolution of the output shear grid, and $d^{\prime}$ is a length scale that depends on the instrument tilt statistics and on the maximum range of valid ADCP measurements. A quadratic fit to the range maxima $\left(r_{\max }\right)-d^{\prime}$ pairs given by Polzin et al. (2002) yields

$$
d^{\prime}=-1.2+0.0857 r_{\max }-0.000136 r_{\max }^{2}
$$

which has an intercept near $r_{\max }=14 \mathrm{~m}$. Estimating $r_{\max } \approx 255 \mathrm{~m}$ as the range at which $80 \%$ of all ensembles have valid velocities yields $d^{\prime} \approx 11.8 \mathrm{~m}$ in case of the dataset considered by Polzin et al. (2002) and here.

As already shown by Polzin et al. (2002), shear spectra based on LADCP data processed with the shear method with interpolation and corrected with transfer function (1) are consistent with the corresponding XCP-based spectra for vertical wavelengths $\geq 50 \mathrm{~m}$, that is, for the entire vertical wavenumber range resolved by the LADCP (red curves in Fig. 2). In spite of the fact that horizontal LADCP velocities associated with small vertical scales are significantly more damped by the shear method with interpolation than by the velocity-inversion method (Fig. 1), it has become fairly common to use a spectral transfer function based on expression (1) to correct shear spectra derived from LADCP profiles processed with the velocityinversion method (e.g., Kunze et al. 2006; MacKinnon et al. 2008; Tian et al. 2009). As illustrated in Fig. 2a (blue curve), in case of the dataset of Polzin et al. (2002) this amounts to a significant overcorrection of the shear spectra for vertical wavelengths up to $\approx 150 \mathrm{~m}$.

Expression (6), which predicts an appropriate length scale to correct for instrument tilting via transfer function (5), is semiempirical and applies strictly only to the dataset of Polzin et al. (2002), which is associated with an rms tilt angle of $\approx 5^{\circ}$. Other LADCP data are associated with significantly different tilt statistics; a survey of recent LADCP datasets collected by this author indicates rms tilt angles ranging from $2^{\circ}$ to $7^{\circ}$. Although suitable replacements for expression (6) can be derived for any dataset, this effort may not be required in practice, at least for LADCP data collected with bin sizes $\leq 10 \mathrm{~m}$ and bin mapping enabled, as the maximum spectral damping associated with instrument tilting in the vertical wavenumber range resolved by the LADCP remains below $10 \%$ in this case (Fig. 3 ).

In case of the processed LADCP velocities used by Polzin et al. (2002), interpolation of the shear measurements onto a uniform depth grid accounts for a significant fraction of the high-wavenumber attenuation. Therefore, the LADCP data were reprocessed with a modified implementation of the shear method that uses simple depth binning, instead of interpolation, to construct the output shear grid. Consistent with expectations, the resulting shear profiles are markedly less damped at short vertical wavelengths than the corresponding 

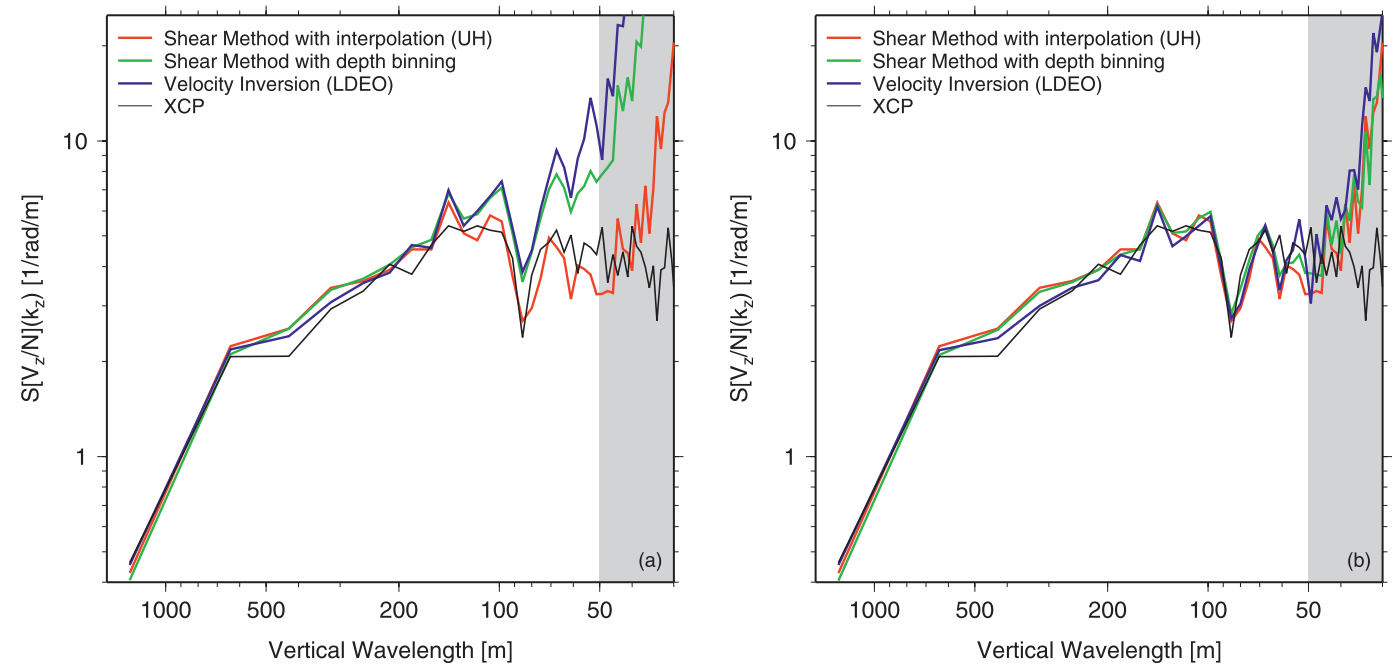

FIG. 2. Corrected vertical wavenumber spectra of buoyancy-frequency-normalized vertical shear between 220 and $1500 \mathrm{~m}$ in the dataset of Polzin et al. (2002); axes, gray shading, and colors are as in Fig. 1. The XCP spectra are corrected for finite-differencing attenuation using expression (3) with $\Delta z_{\mathrm{fd}}=2.5 \mathrm{~m}$. (a) LADCP spectra corrected with transfer function (1) [or, equivalently, with expression (30) of Polzin et al. (2002) with $T_{\mathrm{bsl}}=1$ ]. (b) Spectra corrected with the processing-specific transfer functions described in the text.

profiles derived with the shear method with interpolation (Fig. 1). To correct the resulting spectra, $T_{\text {int }}$ in expression (1) must be replaced by the appropriate transfer function for depth binning,

$$
T_{\text {bin }}\left(k_{z}\right)=\operatorname{sinc}^{2}\left(\frac{k_{z} \Delta z_{g}}{2 \pi}\right) .
$$

[Polzin et al. (2002, p. 213) list the same expression based on $\Delta z_{r}$, rather than $\Delta z_{g}$, which is assumed to by a typographical error.] Using, therefore, the product of expressions (2), (3), (5), and (7) to correct the spectra derived with the shear method with depth binning yields spectral levels that are consistent with the XCP data for all vertical wavelengths resolved by the LADCP (green curve in Fig. 2 b).

The method that is currently most widely used for LADCP processing is the velocity-inversion method of Visbeck (2002). In this method, there is no explicit finite differencing or shear interpolation, but the velocities are usually prebinned into "superensembles" before the inversion and simple depth binning is used to construct the output velocity grid. The appropriate transfer function for correcting shear spectra derived with the velocity-inversion method becomes

$$
T_{\mathrm{VI}}=T_{\mathrm{ra}} \times T_{\text {bin }} \times \operatorname{sinc}^{2}\left(\frac{k_{z} \Delta z_{s}}{2 \pi}\right) \times T_{\text {tilt }},
$$

where $\Delta z_{s}$ is the superensemble preaveraging interval, which is often chosen to be equal to $\Delta z_{g}$. Using expression (8) to correct the LADCP shear spectra of the
Polzin et al. (2002) data processed with the velocityinversion method again yields spectral levels that are consistent with the XCP data for all vertical wavelengths resolved by the LADCP (blue curve in Fig. 2b).

Following Polzin et al. (2002), the results presented so far have been derived exclusively from LADCP downcast data. Although there is no a priori reason why upcastonly data should yield significantly different shear spectra, there is additional (temporal) averaging involved when the downcast and upcast data are combined. [In "standard" LADCP processing, the downcast and upcast data are nearly always combined, primarily in order to use ship drift (GPS) data to constrain the depth-averaged velocities (e.g., Thurnherr 2010).] To

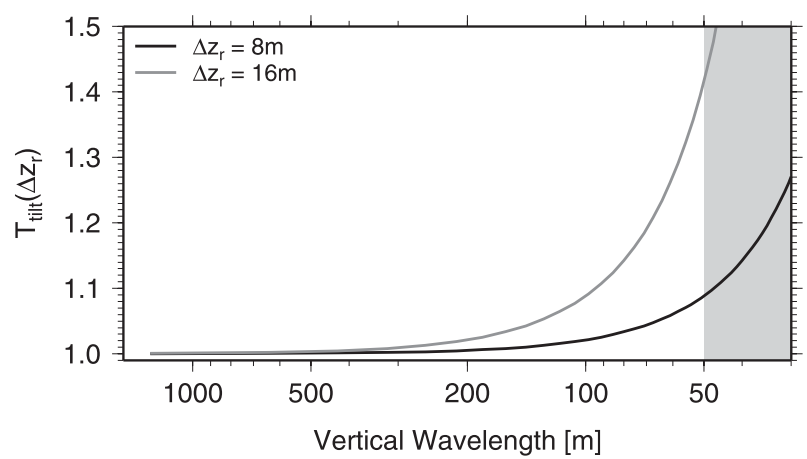

FIG. 3. Worst-case corrections required to compensate verticalshear spectra for instrument-tilting effects for LADCP data collected with bin mapping enabled for typical bin sizes used with 150- and $300-\mathrm{kHz}$ instruments (16 and $8 \mathrm{~m}$, respectively); the wavenumber range not resolved by the LADCP $\left(\lambda_{z} \leq 50 \mathrm{~m}\right)$ is shaded gray. 
TABLE 1. Ratios of the levels of LADCP-derived vs XCPderived shear spectra (as in Fig. 12 of Polzin et al. 2002) in the "resolved finescale band" (50-320-m vertical wavelength).

\begin{tabular}{lccc}
\hline \hline Processing method & Downcast & Upcast & Combined \\
\hline $\begin{array}{l}\text { Shear method with } \\
\text { interpolation (UH) }\end{array}$ & 0.97 & 0.80 & 0.54 \\
$\begin{array}{c}\text { Shear method with } \\
\text { depth binning }\end{array}$ & 1.02 & 1.06 & 0.72 \\
$\begin{array}{l}\text { Velocity inversion } \\
\text { (LDEO) }\end{array}$ & 1.00 & 1.06 & 0.73 \\
\hline
\end{tabular}

test these predictions, additional shear spectra were calculated from the upcast-only and from the combined downcast/upcast LADCP solutions. The resulting ratios of the LADCP- to XCP-derived finescale shear spectra listed in Table 1 confirm that the spectral corrections discussed here bring the LADCP-derived finescale shear levels of the downcast-only and upcast-only solutions into reasonable agreement with the XCP-derived spectra. The corrected shear spectra calculated from the combined downcast/upcast solutions, on the other hand, remain significantly attenuated compared to the XCPbased spectra, regardless of the processing method used.

\section{Discussion}

The results presented above indicate that different processing methods can be used to estimate shear levels from LADCP data over the entire range of resolved vertical scales, as long as the LADCP shear is derived from downcast-only or upcast-only profiles and as long as appropriate transfer functions are used to correct the spectra. Using the wrong spectral corrections, on the other hand, yields biased finescale shear levels, in particular at short vertical wavelengths. Whether the resulting biases are significant depends sensitively on the wavenumber range that is used in the subsequent analysis: based on the dataset considered here, the erroneous spectral correction used by Kunze et al. $(2006)^{1}$ for vertical wavelengths $\geq 150 \mathrm{~m}$ will lead to significantly less bias in the resulting turbulence and mixing estimates than the same correction used, for example, by MacKinnon et al. (2008) and by Tian et al. (2009) with vertical cutoff wavelengths between 50 and $90 \mathrm{~m}$. It can be noted, however, that part of the shear-level high bias resulting from applying the wrong transfer function in all these cited studies is compensated by the fact that no range-dependent corrections were applied.

\footnotetext{
${ }^{1}$ Only a small subset of the LADCP sections used by Kunze et al. (2006) was processed with the velocity-inversion method; for the remainder, the correct spectral transfer function was used.
}

Although the suitably corrected shear spectra in the dataset considered here are essentially identical regardless of the LADCP processing method used, it may be worth reiterating that the velocity-inversion software causes the least attenuation and therefore requires the smallest spectral corrections of all methods considered here. The attenuation associated with velocity-inversion processing can be further reduced by decreasing $\Delta z_{s}$, that is, by shortening the length of the superensembles. Given sufficient memory, it is even possible to disable preaveraging altogether. In case of the dataset considered here, there is no significant difference between the corrected spectral levels at the resolved vertical scales derived with the velocity-inversion method, regardless of whether preaveraging is used or whether the data are processed at 16- or 5-m vertical resolution (not shown).

Acknowledgments. Funding for the analysis presented here was provided by the National Science Foundation under Grant OCE-1030309. The CTD/LADCP data of Polzin et al. (2002) were graciously provided by the authors of that study. Helpful comments on an early manuscript draft by E. Firing and E. Kunze are gratefully acknowledged.

\section{REFERENCES}

Firing, E., and R. Gordon, 1990: Deep ocean acoustic Doppler current profiling. Proc. Fourth Working Conf. on Current Measurements, Clinton, MD, IEEE, 192-201.

Fischer, J., and M. Visbeck, 1993: Deep velocity profiling with self-contained ADCPs. J. Atmos. Oceanic Technol., 10, 764 773.

King, B. A., E. Firing, and T. M. Joyce, 2001: Shipboard observations during WOCE. Ocean Circulation \& Climate, G. Siedler, J. Church, and J. Gould, Eds., Academic Press, 99-122.

Kunze, E., E. Firing, J. M. Hummon, T. K. Chereskin, and A. M Thurnherr, 2006: Global abyssal mixing inferred from lowered ADCP shear and CTD strain profiles. J. Phys. Oceanogr., 36, 1553-1576.

MacKinnon, J. A., T. M. S. Johnston, and R. Pinkel, 2008: Strong transport and mixing of deep water through the southwest Indian Ridge. Nat. Geosci., 1, 755-758.

Naveira-Garabato, A. C., K. L. Polzin, B. A. K. King, K. J. Heywood, and M. Visbeck, 2004: Widespread intense turbulent mixing in the Southern Ocean. Science, 303, 210-213.

Polzin, K., E. Kunze, J. Hummon, and E. Firing, 2002: The finescale response of lowered ADCP velocity profiles. J. Atmos. Oceanic Technol., 19, 205-224.

Thurnherr, A. M., 2010: A practical assessment of uncertainties in full-depth velocity profiles obtained with Teledyne/RDI Workhorse acoustic Doppler current profilers. J. Atmos. Oceanic Technol., 27, 1215-1227.

Tian, J., Q. Yang, and W. Zhao, 2009: Enhanced diapycnal mixing in the South China Sea. J. Phys. Oceanogr., 39, 3191-3203.

Visbeck, M., 2002: Deep velocity profiling using lowered acoustic Doppler current profilers: Bottom track and inverse solutions. J. Atmos. Oceanic Technol., 19, 794-807. 